\title{
Lacunary Statistical Convergence of Sequences of Real-Valued Functions
}

\begin{abstract}
A. Gökhan
Department of Secondary Science and Mathematics Education, Firat University, 23119 Elaziğ, Turkey

Correspondence should be addressed to A. Gökhan; agokhan1@firat.edu.tr

Received 10 September 2012; Accepted 26 November 2012

Academic Editor: Peijun Guo

Copyright (C) 2013 A. Gökhan. This is an open access article distributed under the Creative Commons Attribution License, which permits unrestricted use, distribution, and reproduction in any medium, provided the original work is properly cited.

We introduce the concepts of the lacunary statistical convergence of sequences of real-valued functions. We also give the relation between this convergence and strongly lacunary and pointwise statistical convergence. Furthermore we introduce the concept of a lacunary statistical Cauchy sequence for functional sequences and prove that it is equivalent to lacunary statistical convergence of sequences of real-valued functions.
\end{abstract}

\section{Introduction}

The idea of the statistical convergence of sequence of real and complex numbers was introduced by Fast [1] and also independently by Buck [2] and Shoenberg [3]. Gökhan and Güngör [4] defined pointwise statistical convergence of sequences of real-valued functions by saying that st $\lim f_{k}(x)=f(x)$ or $f_{k} \stackrel{\text { st. }}{\longrightarrow} f$ on $S$ if and only if for every $\varepsilon>0$,

$$
\lim _{n \rightarrow \infty} \frac{1}{n} \mid\left\{k \leq n:\left|f_{k}(x)-f(x)\right| \geq \varepsilon \text { for every } x \in S\right\} \mid=0 \text {. }
$$

By a lacunary sequence we mean an increasing integer sequence $\theta=\left(k_{r}\right)$ such that $k_{0}=0$ and $h_{r}=k_{r}-k_{r-1} \rightarrow \infty$ as $r \rightarrow \infty$. Throughout this paper the intervals determined by $\theta$ will be denoted by $I_{r}=\left(k_{r-1}, k_{r}\right]$ and the ratio $k_{r} / k_{r-1}$ will be abbreviated by $q_{r}$.

Quite recently Gökhan et al. [5] defined the strongly lacunary convergence of sequences of real-valued functions in the following way.

For any lacunary sequence $\theta=\left(k_{r}\right)$, a sequence of functions $\left(f_{k}\right)$ is said to be strongly lacunary convergent to $f$ on the set $S \subset \mathbb{R}$ if there exists $f(x)$ such that

$$
\frac{1}{h_{r}} \sum_{I_{r}}\left|f_{k}(x)-f(x)\right| \longrightarrow 0
$$

for every $x \in S$. We denote this symbolically by writing $f_{k} \stackrel{N_{\theta}}{\longrightarrow} f$ on $S$.

Fridy and Orhan [6] defined lacunary statistical convergence by saying that $S_{\theta}-\lim x=L$ or $x_{k} \rightarrow L\left(S_{\theta}\right)$ if and only if for every $\varepsilon>0$,

$$
\lim _{r} \frac{1}{h_{r}}\left|\left\{k \in I_{r}:\left|x_{k}-L\right| \geq \varepsilon\right\}\right|=0 .
$$

Furthermore, Fridy and Orhan [7] introduced the lacunary statistical Cauchy sequence.

\section{Lacunary Statistical Convergence}

Now, we introduce the concepts of the lacunary statistical convergence of sequences of real valued functions.

Definition 1. Let $\theta$ be a lacunary sequence, and the sequence $\left(f_{k}\right)$ is $\theta$-lacunary statistically convergent to $f(x)$ on $S$ provided that for every $\varepsilon>0$,

$$
\lim _{r} \frac{1}{h_{r}} \mid\left\{k \in I_{r}:\left|f_{k}(x)-f(x)\right| \geq \varepsilon \text { for every } x \in S\right\} \mid=0 .
$$

In this case we write $S_{\theta}-\lim f_{k}(x)=f(x)$ or $f_{k} \stackrel{S_{\theta}}{\rightarrow} f$ on $S$. 
Now, we first give some relations between strongly lacunary convergence and lacunary stratistical convergence and show that they are equivalent for bounded sequences. We also study $f_{k} \stackrel{\text { st. }}{\longrightarrow} f$ on $S \Rightarrow f_{k} \stackrel{S_{\theta}}{\longrightarrow} f$ on $S$ and $f_{k} \stackrel{S_{\theta}}{\longrightarrow} f \Rightarrow f_{k} \stackrel{\text { st. }}{\longrightarrow}$ $f$ on $S$ under certain restrictions on $\theta=\left(k_{r}\right)$.

Theorem 2. Let $\theta=\left(k_{r}\right)$ be a lacunary sequence; then

(i) $f_{k} \stackrel{N_{\theta}}{\longrightarrow} f$ implies $f_{k} \stackrel{S_{\theta}}{\longrightarrow} f$ on $S$;

(ii) $\sup _{k}\left|f_{k}(x)\right|<\infty$ for every $x \in S$ and $f_{k} \stackrel{S_{\theta}}{\rightarrow} f$ implies $f_{k} \stackrel{N_{\theta}}{\longrightarrow} f$ on $S$;

(iii) let $\sup _{k}\left|f_{k}(x)\right|<\infty$ for every $x \in S$. Then $f_{k} \stackrel{S_{\theta}}{\longrightarrow} f$ if and only if $f_{k} \stackrel{N_{\theta}}{\longrightarrow} f$ on $S$.

Proof. (i) If $\varepsilon>0$ and $f_{k} \stackrel{N_{\theta}}{\longrightarrow} f$ on $S$ we can write

$$
\begin{aligned}
\sum_{k \in I_{r}}\left|f_{k}(x)-f(x)\right| & \\
& \geq \sum_{\substack{k \in I_{r} \\
\left|f_{k}(x)-f(x)\right| \geq \varepsilon}}\left|f_{k}(x)-f(x)\right| \\
& \geq \varepsilon \mid\left\{k \in I_{r}:\left|f_{k}(x)-f(x)\right| \geq \varepsilon \text { for every } x \in S\right\} \mid
\end{aligned}
$$

which yields the result.

(ii) Suppose that $f_{k} \stackrel{S_{\theta}}{\rightarrow} f$ and $\sup _{k}\left|f_{k}(x)\right|<\infty$ on $S$, say

$$
\left|f_{k}(x)-f(x)\right| \leq M_{x}
$$

for all $k$ and every $x \in S$. Given $\varepsilon>0$ we get

$$
\begin{aligned}
& \frac{1}{h_{r}} \sum_{k \in I_{r}}\left|f_{k}(x)-f(x)\right| \\
& =\frac{1}{h_{r}} \sum_{\substack{k \in I_{r} \\
\left|f_{k}(x)-f(x)\right| \geq \varepsilon}}\left|f_{k}(x)-f(x)\right| \\
& \quad+\frac{1}{h_{r}} \sum_{k \in I_{r}}\left|f_{k}(x)-f(x)\right| \\
& \leq \frac{M_{x}}{h_{r}} \mid\left\{k \in I_{k}(x)-f(x) \mid<\varepsilon\right.
\end{aligned}
$$

for every $x \in S$, from which the result follows.
Let $\theta$ be given and define $f_{k}(x)$ to be $|x|, 2|x|, \ldots,\left[\sqrt{h_{r}}\right]$. $|x|$ at the first $\left[\sqrt{h_{r}}\right]$ integers in $I_{r}$, and $x_{k}=0$ otherwise on $\mathbb{R}$. Note that $\left(f_{k}\right)$ is not bounded. We have, for every $\varepsilon>0$,

$$
\begin{gathered}
\frac{1}{h_{r}} \mid\left\{k \in I_{r}:\left|f_{k}(x)-0\right| \geq \varepsilon \text { for every } x \in \mathbb{R}\right\} \mid \\
=\frac{\left[\sqrt{h_{r}}\right]}{h_{r}} \longrightarrow 0, \quad \text { as } r \longrightarrow \infty
\end{gathered}
$$

that is, $f_{k} \stackrel{S_{\theta}}{\rightarrow} f=0$. On the other hand,

$$
\begin{aligned}
& \frac{1}{h_{r}} \sum_{k \in I_{r}}\left|f_{k}(x)-0\right| \\
& \quad=\frac{1}{h_{r}} \frac{\left[\sqrt{h_{r}}\right]\left(\left[\sqrt{h_{r}}\right]+1\right) \cdot|x|}{2} \longrightarrow \frac{|x|}{2} \neq 0
\end{aligned}
$$

on $\mathbb{R}-\{0\}$; hence $f_{k} \stackrel{N_{\theta}}{\rightarrow} f=0$.

We remark that the example given in (ii) shows that the boundedness condition cannot be omitted from the hypothesis of Theorem 2(ii).

(iii) This is an immediate consequence of (i) and (ii).

Lemma 3. For any lacunary sequence $\theta, f_{k} \stackrel{\text { st. }}{\rightarrow} f$ implies $f_{k} \stackrel{S_{\theta}}{\rightarrow} f$ on $S$ if and only if $\liminf _{r} q_{r}>1$.

Proof. Suppose first that $\liminf _{r} q_{r}>1$; then there exists a $\delta>0$ such that $q_{r} \geq 1+\delta$ for sufficiently large $r$, which implies that

$$
\frac{h_{r}}{k_{r}} \geq \frac{\delta}{1+\delta}
$$

If $f_{k} \stackrel{\text { st. }}{\longrightarrow} f$ on $S$, then for every $\varepsilon>0$ and for sufficiently large $r$, we have

$$
\begin{aligned}
& \frac{1}{k_{r}} \mid\left\{k \leq k_{r}:\left|f_{k}(x)-f(x)\right| \geq \varepsilon \text { for every } x \in S\right\} \mid \\
& \quad \geq \frac{1}{k_{r}} \mid\left\{k \in I_{r}:\left|f_{k}(x)-f(x)\right| \geq \varepsilon \text { for every } x \in S\right\} \mid \\
& \quad \geq \frac{\delta}{1+\delta} \frac{1}{h_{r}} \mid\left\{k \in I_{r}:\left|f_{k}(x)-f(x)\right| \geq \varepsilon \text { for every } x \in S\right\} \mid .
\end{aligned}
$$

This proves the sufficiency.

Conversely, suppose that $\liminf _{r} q_{r}>1$. Proceeding as in Lemma 2.1 [5] we can select a subsequence $\left(k_{r(j)}\right)$ of the lacunary sequence $\theta=\left(k_{r}\right)$ such that

$$
\frac{k_{r(j)}}{k_{r(j)-1}}<1+\frac{1}{j}, \quad \frac{k_{r(j)-1}}{k_{r(j-1)}}>j,
$$

where $r(j) \geq r(j-1)+2$. 
Now define a bounded sequence $\left(f_{k}\right)$ by $f_{i}(x)=x$ if $i \in I_{r(j)}$ for some $j=1,2, \ldots$ and $f_{i}(x)=0$ otherwise on $\mathbb{R}$. It is shown in Lemma 2.1 [5] that $\left(f_{k}\right)$ is not strongly lacunarily convergent on $\mathbb{R}$, but $\left(f_{k}\right)$ is strongly Cesáro summability. Theorem 2(ii) implies that $\left(f_{k}\right)$ is not lacunarily statistically convergent, but it follows from Theorem 2.3. (i) of [5] that $\left(f_{k}\right)$ is pointwise statistically convergent on $S$. Hence we obtain that $\left(f_{k}\right)$ is pointwise statistical convergent on $S$, but it is not lacunarily statistically convergent on $S$. This contradicts the assumption, whence $\lim _{\inf _{r}} q_{r}>1$.

Lemma 4. For any lacunary sequence $\theta, f_{k} \stackrel{S_{\theta}}{\longrightarrow} f$ on $S$ implies $f_{k} \stackrel{\text { st. }}{\longrightarrow} f$ on $S$ if and only if $\lim \sup _{r} q_{r}<\infty$.

Proof. If limsup $q_{r}<\infty$, then there is an $H>0$ such that $q_{r}<H$ for all $r$. Suppose that $f_{k} \stackrel{S_{\theta}}{\longrightarrow} f$ on $S$, and let

$$
N_{r, x}=\mid\left\{k \in I_{r}:\left|f_{k}(x)-f(x)\right| \geq \varepsilon \text { for every } x \in S\right\} \mid .
$$

By (4) given $\varepsilon>0$, there is an $r_{0} \in \mathbb{N}$ such that

$$
\frac{N_{r, x}}{h_{r}}<\varepsilon \text { for all } r>r_{0} \text { and every } x \in S \text {. }
$$

Now let $M_{x}=\left\{\max N_{r, x}: 1 \leq r \leq r_{0}\right\}$ for every $x \in S$ and let $n$ be any integer satisfying $k_{r-1}<n \leq k_{r}$; then we can write

$$
\begin{aligned}
\frac{1}{n} \mid & \left\{k \leq n:\left|f_{k}(x)-f(x)\right| \geq \varepsilon \text { for every } x \in S\right\} \mid \\
& \leq \frac{1}{k_{r-1}} \mid\left\{k \leq k_{r}:\left|f_{k}(x)-f(x)\right| \geq \varepsilon \text { for every } x \in S\right\} \mid \\
& =\frac{1}{k_{r-1}}\left\{N_{1, x}+N_{2, x}+\cdots+N_{r_{0}, x}+N_{r_{0}+1, x}+\cdots+N_{r, x}\right\} \\
& \leq \frac{M_{x}}{k_{r-1}} \cdot r_{0}+\frac{1}{k_{r-1}}\left\{h_{r_{0}+1} \frac{N_{r_{0}+1, x}}{h_{r_{0}+1}}+\cdots+h_{r} \frac{N_{r, x}}{h_{r}}\right\} \\
& \leq \frac{r_{0} \cdot M_{x}}{k_{r-1}}+\frac{1}{k_{r-1}}\left(\sup _{r>r_{0}} \frac{N_{r, x}}{h_{r}}\right)\left\{h_{r_{0}+1}+\cdots+h_{r}\right\} \\
& \leq \frac{r_{0} \cdot M_{x}}{k_{r-1}}+\varepsilon \cdot \frac{k_{r}-k_{r_{0}}}{k_{r-1}} \text { by }(14) \\
& \leq \frac{r_{0} \cdot M_{x}}{k_{r-1}}+\varepsilon \cdot q_{r} \leq \frac{r_{0} \cdot M_{x}}{k_{r-1}}+\varepsilon H
\end{aligned}
$$

for every $x \in S$, and the sufficiency follows immediately.

Conversely, suppose that $\lim \sup _{r} q_{r}=\infty$. Following the idea in Lemma 2.2 [5] we can select a subsequence $\left(k_{r(j)}\right)$ of the lacunary sequence $\theta=\left(k_{r}\right)$ such that $q_{r(j)}>j$, and define a bounded sequence by $f_{i}(x)=x$ if $k_{r(j)-1}<i \leq 2 k_{r(j)-1}$ for some $j=1,2, \ldots$, and $f_{i}(x)=0$ otherwise on $\mathbb{R}$. It is shown in Lemma 2.2 [5] that $f_{k} \stackrel{N_{\theta}}{\longrightarrow} f=0$ on $\mathbb{R}$ but $f_{k} \stackrel{\left|\sigma_{1}\right|}{\rightarrow} f$ on $\mathbb{R}$. By Theorem 2(i) we conclude that $f_{k} \stackrel{s_{\theta}}{\rightarrow} f=0$ on $\mathbb{R}$ but
Theorem 2.3 of [5] implies that $f_{k} \stackrel{\text { st. }}{\rightarrow} f=0$ on $\mathbb{R}$. Hence $f_{k} \stackrel{S_{\theta}}{\rightarrow} f=0$ does not imply $f_{k} \stackrel{\text { st. }}{\longrightarrow} f=0$ on $\mathbb{R}$.

Combining Lemmas 3 and 4 we get the following.

Theorem 5. Let $\theta$ be a lacunary sequence and let $\left(f_{k}\right)$ be a sequence offunctions on a set $S$; then $f_{k} \stackrel{\text { st. }}{\rightarrow} f$ and $f_{k} \stackrel{S_{\theta}}{\longrightarrow} f$ if and only if

$$
1<\lim \inf _{r} q_{r} \leq \lim \sup _{r} q_{r}<\infty
$$

\section{Cauchy Criterion}

Now we introduce the lacunary statistical analog of the Cauchy convergence criterion which is, as we shall see, equivalent to the lacunary statistical convergence.

Definition 6. Let $\theta=\left(k_{r}\right)$ be a lacunary sequence and let $\left(f_{k}\right)$ be a sequence of functions on a set $S$. The sequence $\left(f_{k}\right)$ is said to be a $\theta$ lacunary statistical Cauchy sequence if there is a subsequence $\left(f_{k^{\prime}(r)}\right)$ of $\left(f_{k}\right)$ such that $k^{\prime}(r) \in I_{r}$ for each $r$, $\lim _{r} f_{k_{(r)}^{\prime}}(x)=f(x)$ on $S$, and for every $\varepsilon>0$

$\lim _{r} \frac{1}{h_{r}} \mid\left\{k \in I_{r}:\left|f_{k}(x)-f_{k^{\prime}(r)}(x)\right| \geq \varepsilon\right.$ for every $\left.x \in S\right\} \mid=0$.

Theorem 7. The sequence $\left(f_{k}\right)$ is lacunarily statistically convergent if and only if $\left(f_{k}\right)$ is a lacunary statistical Cauchy sequence.

Proof. Let $f_{k} \stackrel{S_{\theta}}{\longrightarrow} f$ on $S$, and write

$$
K_{x}^{(j)}=\left\{k \in \mathbb{N}:\left|f_{k}(x)-f(x)\right|<\frac{1}{j} \text { for every } x \in S\right\}
$$

for each $j \in \mathbb{N}$. Hence, for each $j, K_{x}^{(j)} \supseteq K_{x}^{(j+1)}$ for every $x \in S$ and

$$
\frac{\left|K_{x}^{(j)} \cap I_{r}\right|}{h_{r}} \longrightarrow 1 \text { as } r \longrightarrow \infty .
$$

Choose $m(1)$ such that $r \geq m(1)$ implies $\left|K_{x}^{(1)} \cap I_{r}\right| / h_{r}>$ 0 , that is, $K_{x}^{(1)} \cap I_{r} \neq \phi$ for every $x \in S$. Next choose $m(2)>$ $m(1)$ so that $r \geq m(2)$ implies $K_{x}^{(2)} \cap I_{r} \neq \phi$ for every $x \in S$. Then for each $r$ satisfying $m(1) \leq r<m(2)$, choose $k^{\prime}(r) \in I_{r}$ such that $k^{\prime}(r) \in I_{r} \cap K_{x}^{(1)}$, that is, $\left|f_{k^{\prime}(r)}(x)-f(x)\right|<1$ for every $x \in S$. In general, choose $m(p+1)>m(p)$ such that $r>m(p+1)$ implies $I_{r} \cap K_{x}^{(p+1)} \neq \phi$ for every $x \in S$. Then for all $r$ satisfying $m(p) \leq r<m(p+1)$, choose $k^{\prime}(r) \in I_{r} \cap K_{x}^{(p)}$ for every $x \in S$, that is:

$$
\left|f_{k^{\prime}(r)}(x)-f(x)\right|<\frac{1}{p}
$$


for every $x \in S$. Hence, we get $k^{\prime}(r) \in I_{r}$ for every $r$, and (20) implies that $\lim _{r} f_{k^{\prime}(r)}(x)=f(x)$ for every $x \in S$. Furthermore, we have, for every $\varepsilon>0$,

$$
\begin{aligned}
\frac{1}{h_{r}} \mid\left\{k \in I_{r}:\left|f_{k}(x)-f_{k^{\prime}(r)}(x)\right| \geq \varepsilon \text { for every } x \in S\right\} \mid \\
\leq \frac{1}{h_{r}} \mid\left\{k \in I_{r}:\left|f_{k}(x)-f(x)\right| \geq \frac{\varepsilon}{2} \text { for every } x \in S\right\} \mid \\
\quad+\frac{1}{h_{r}} \mid\left\{k \in I_{r}:\left|f_{k^{\prime}(r)}(x)-f(x)\right| \geq \frac{\varepsilon}{2} \text { for every } x \in S\right\} \mid .
\end{aligned}
$$

Using the assumption that $f_{k} \stackrel{S_{\theta}}{\longrightarrow} f$ on $S$ and $\lim _{r} f_{k^{\prime}(r)}(x)=$ $f(x)$ on $S$, we infer (17), whence $\left(f_{k}(x)\right)$ is a $\theta$ lacunary statistical Cauchy sequence on $S$.

Conversely, suppose that $\left(f_{k}(x)\right)$ is a $\theta$ lacunary statistical Cauchy sequence. For every $\varepsilon>0$, we have

$$
\begin{aligned}
\mid\{k \in & \left.I_{r}:\left|f_{k}(x)-f(x)\right| \geq \varepsilon \text { for every } x \in S\right\} \mid \\
\leq & \mid\left\{k \in I_{r}:\left|f_{k}(x)-f_{k^{\prime}(r)}(x)\right| \geq \frac{\varepsilon}{2} \text { for every } x \in S\right\} \mid \\
& +\mid\left\{k \in I_{r}:\left|f_{k^{\prime}(r)}(x)-f(x)\right| \geq \frac{\varepsilon}{2} \text { for every } x \in S\right\} \mid,
\end{aligned}
$$

from which it follows that $f_{k} \stackrel{S_{\theta}}{\rightarrow} f$ on $S$.

\section{Conclusion}

It is know that several different types of convergence may define sequences $\left(f_{k}\right)$ of which trems are real-valued functions having a common domain on the real line $\mathbb{R}$. The present paper gives the lacunary statistical convergence of a sequence of real-valued functions $\left(f_{k}\right)$ and gives some relations between this convergence and strongly lacunary convergence and shows that they are equivalent for a bounded sequence of real-valued functions. Furthermore, the paper proves that this convergence is equivalent to lacunary statistical Cauchy sequence.

\section{References}

[1] H. Fast, "Sur la convergence statistique," Colloquium Mathematicum, vol. 2, pp. 241-244, 1951.

[2] R. C. Buck, "Generalized asymptotic density," American Journal of Mathematics, vol. 75, no. 2, pp. 335-346, 1953.

[3] I. J. Schoenberg, "The integrability of certain functions and related summability methods," The American Mathematical Monthly, vol. 66, no. 5, pp. 361-375, 1959.

[4] A. Gökhan and M. Güngör, "On pointwise statistical convergence," Indian Journal of Pure and Applied Mathematics, vol. 33, no. 9, pp. 1379-1384, 2002.

[5] A. Gökhan, M. Güngör, and Y. Bulut, "On the strong lacunaryconvergence and strong cesáro summability of sequences of real-valued functions," Applied Sciences, vol. 8, pp. 70-77, 2006.
[6] J. A. Fridy and C. Orhan, "Lacunary statistical convergence," Pacific Journal of Mathematics, vol. 160, no. 1, pp. 43-51, 1993.

[7] J. A. Fridy and C. Orhan, "Lacunary statistical summability," Journal of Mathematical Analysis and Applications, vol. 173, no. 2, pp. 497-504, 1993. 


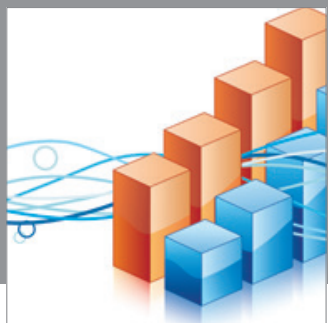

Advances in

Operations Research

mansans

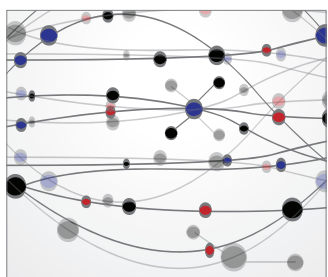

The Scientific World Journal
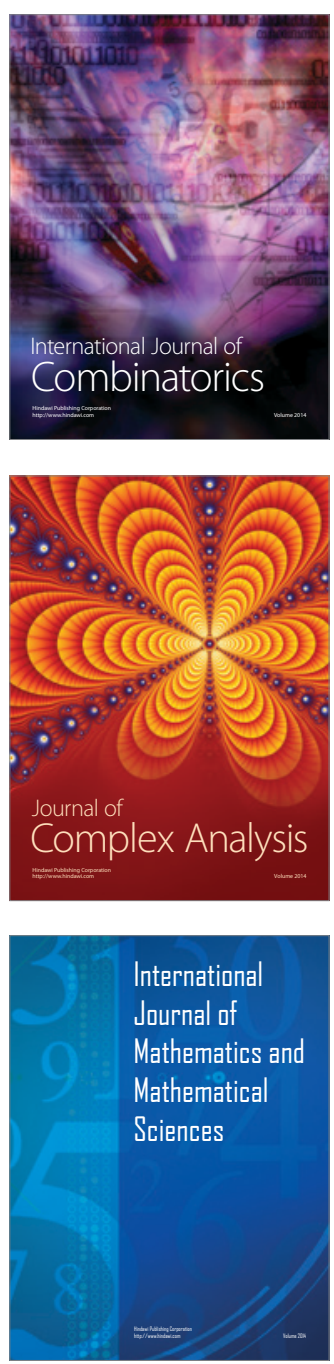
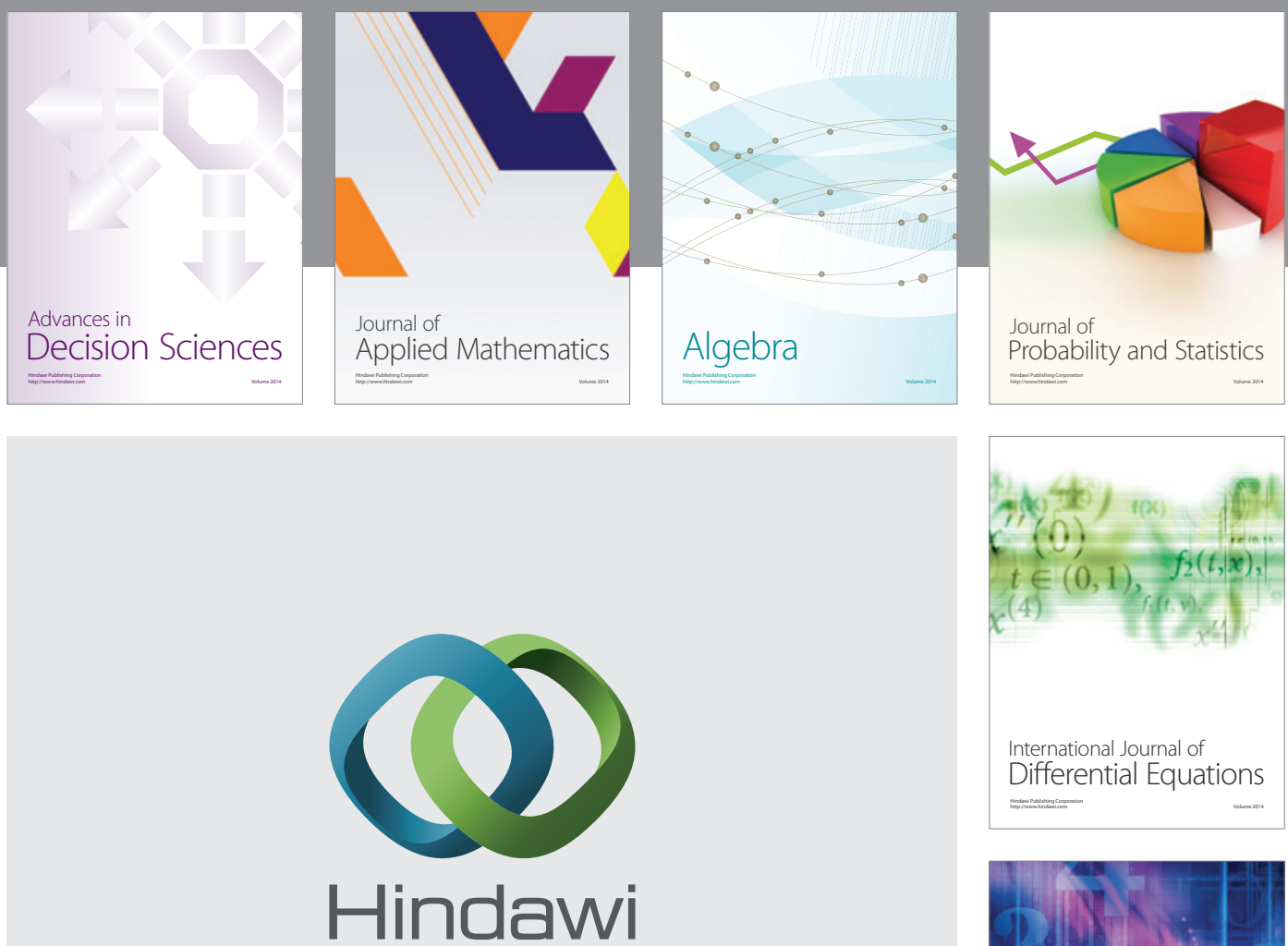

Submit your manuscripts at http://www.hindawi.com
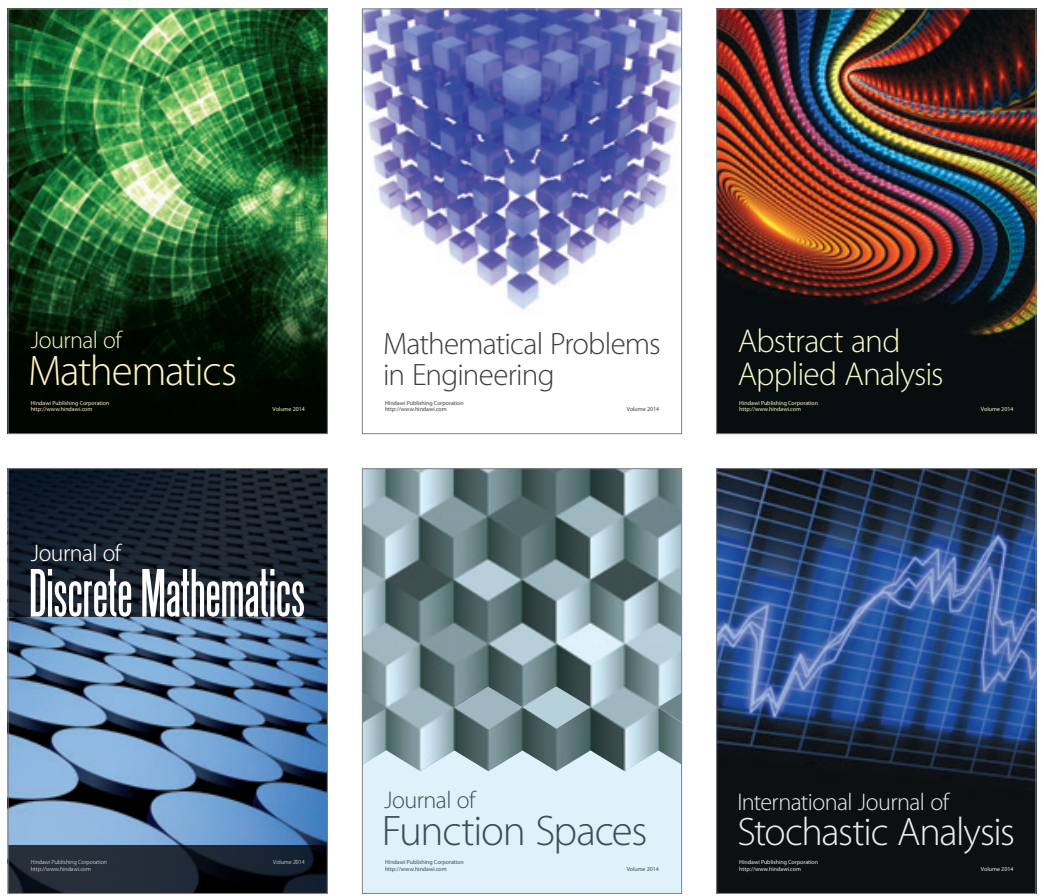

Journal of

Function Spaces

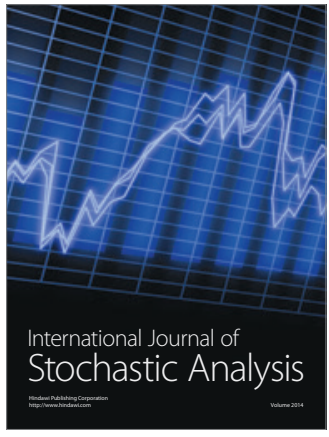

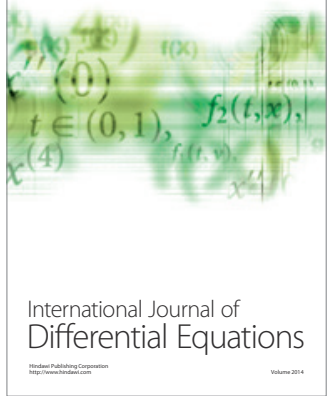
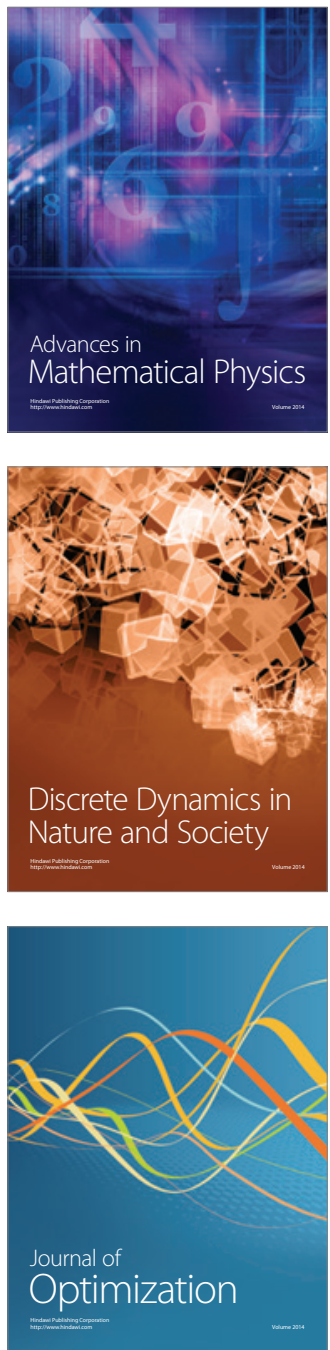